\title{
БАЗОВАЯ СИСТЕМА АВТОМАТИЧЕСКОГО УПРАВЛЕНИЯ ПРОЦЕССОМ ЭКСТРУДИРОВАНИЯ БИОПОЛИМЕРОВ, ЕЕ ОСОБЕННОСТИ, ЗАДАЧИ ПАРАМЕТРИЧЕСКОГО СИНТЕЗА И АНАЛИЗА
}

\author{
Хобин В.А., Егоров В.Б. \\ Одесская национальная академия пищевых технологий
}

\begin{abstract}
Аннотация: Для разработки (синтеза и анализа) и реализации действующего варианта САУ процесса ЭБП было введено понятие ее базового варианта. Он ориентирован решать в автоматическом режиме наиболее динамичные задачи управления, сохранив за оператором и лабораторией управление качеством экструдатов. Разработка САУ, реализующих эти задачи, сделала необходимым проведение структурного и параметрического синтеза системы, анализа ее функционирования, адекватно отражающего условия ее будущей эксплуатации.
\end{abstract}

Ключевые слова: идентификация, математические модели, система автоматического управления, экструдирование, биополимеры.

Ранее, в рамках разработки концепции построения САУ процессом ЭБП, была обоснована перспективная функциональная организация САУ, и разработаны три, альтернативных по использованию управляющего воздействия $u_{9}$, варианта структурных схем для ее реализации. Для реализации выбран вариант, из которого исключена функция измерения косвенных показателей качества экструдата в реальном времени. Принципиальной особенностью такого варианта САУ является то, что использование $\mathrm{u}_{э}$ доступно только в режиме настройки экструдера на работу с заданным видом сырья. Эта настройка осуществляется «вручную», совместно оператором экструдера и лаборантом, и остается, как правило, неизменной, uэ = const, в течение всего времени производства

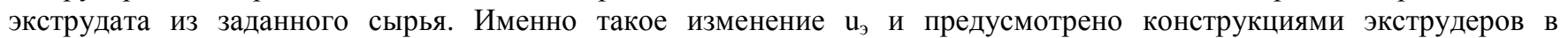
настоящее время. Кроме того, поскольку из базового варианта САУ исключена функция измерения косвенных показателей качества экструдата, то базовой САУ, не реализуется и, связанная с этим измерением, функция гарантирования качественных показателей экструдата. В таком случае, реализация функции управления по гарантированию качественных показателей экструдата возлагается на оператора экструдера и лаборанта.

Важно отметить, что базовая САУ включает в себя наиболее динамичные контура управления. Для заданной структуры САУ, обеспечение их эффективного функционирования требует обоснованного выбора настроечных параметров всех элементов управления [1 - 3]. К этим элементам относятся все шесть регуляторов, а также модули оценки вероятностных характеристик и расчета допустимого заданного значения степени нагрева ПЭД экструдера. Наиболее эффективным инструментом такого выбора настроечных параметров является оптимальный параметрический синтез [4 - 6]. Разработанная имитационная модель процесса ЭБП [7, 8] позволяет реализовать процедуру синтеза алгоритмическими методами, в ходе специальным образом организованных машинных экспериментов с имитационной моделью САУ [9 - 12].

На структурной схеме базовой САУ, для подсистемы регулирования температурного режима тепловой обработки сырья при его движении вдоль оси шнекового пресса, априори выбрана каскадная структура. Вместе с тем, в предыдущих публикациях были рассмотрены три различных варианта структурных решений для этой подсистемы. При этом важно подчеркнуть, что эти варианты структурных решений могут реализовываться вне зависимости от выбранного варианта использования $\mathrm{u}_{3}$, поэтому целесообразно провести сравнительный анализ этих вариантов и подтвердить, или опровергнуть, правильность принятого решения. Этому анализу должен предшествовать оптимальный параметрический синтез регуляторов подсистемы.

Кроме того, целесообразно провести сравнительный анализ эффективности функционирования САУ, при различных вариантах алгоритмов автоматического управления, с управлением процессом ЭБП в «ручном» режиме. Очевидно, что такое сравнение будет более объективным, когда его результаты получены методом моделирования, т.к. в реальных условиях обеспечить их тождественность для различных экспериментов невозможно. Кроме того, моделирование ручного режима управления даст возможность устранить субъективный фактор, который неизбежно привносится оператором при реализации им процедуры управления. 
Параметрический синтез и анализ подсистем регулирования с типовыми алгоритмами. Для типового ПИДалгоритма регулирования выражение алгоритма регулирования тока нагрузки ПЭД экструдера, реализующего принцип замкнутого управления, принимает вид:

$$
u_{n}(t)=k_{p_{I}}\left(\Delta I_{n \ni \partial}(t)+\frac{1}{T_{u з I}} \int_{0}^{t} \Delta I_{n \ni \partial}(t) d t+T_{\text {прг }} \frac{d \Delta I_{n \ni \partial}(t)}{d t}\right)+u_{n_{0}},
$$

где $u_{n_{0}}$ - начальное значение управляющего воздействия $\mathrm{u}_{\text {п }}(\mathrm{t})$ в момент замыкания контура регулирования, обеспечивающее «безударность» этого замыкания;

$\Delta I_{n \ni \partial}(t)=I_{n \ni \partial}^{\text {здд+ }}(t)-I_{n \ni \partial}(t)$ - ошибка регулирования тока нагрузки $\mathrm{I}(\mathrm{t}) ;$

$I_{n \ni \partial}^{3 д \partial+}(t)$ - заданное значение тока нагрузки, с учетом гарантирования соблюдения теплового режима ПЭд (ограничения «сверху» на величину перегрева ПЭД экструдера);

$k_{p I}, T_{\text {изI }}, T_{\text {прI }}$ - настроечные параметры регулятора тока, подлежащие оптимизации.

Оптимизация осуществлялась по интегральному квадратичному критерию в соответствии с выражением (1.2), в ходе специального компьютерного эксперимента с использованием имитационной модели подсистемы. При этом время моделирования $\mathrm{T}_{\text {мод }} \mathrm{CAУ}$ на каждом шаге оптимизации соответствовало времени, на котором осуществлялось моделирование возмущений, приведенных к каналам изменения напряжения питающей сети и влажности экструдируемого сырья.

$$
k_{p I}^{*}, T_{u з I}^{*}, T_{n p I}^{*}=\operatorname{argmin}\left\{\int_{0}^{T_{\text {мод }}}\left(I_{n \ni \partial}^{3 \partial \partial+}-I_{n \ni \partial}\left(k_{p I}, T_{u з I}, T_{n p I}, t\right)\right)^{2} d t\right\},
$$

Значения начальных приближений $\mathrm{k}_{\mathrm{pI}}, T_{\text {из }}, T_{\text {прI }}$ настроечных параметров для реализации первого шага поисковой процедуры оптимизации (1.2) найдены исходя из свойств типовой модели динамики по каналу регулирования, в соответствии с рекомендациями [4-6]: $k_{p I}=0,85 \%$ у.в./А, $T_{u з I}=7 \mathrm{c}, T_{\text {прI }}=3$ с. 3 начения параметров, найденные в ходе процедуры оптимизации и округленные: $k_{p I}^{*}=1,0 \%$ у.в./А, $T_{u з I}^{*}=8,0$ с, $T_{n p I}^{*}=2,0$ c.

Параметрический синтез альтернативных вариантов подсистемы регулирования температурного режима обработки сырья в экструдере. Вариант подсистемы, реализуемый на основе замкнутого принципа управления с «классической» структурой, когда значения температур $\theta_{\mathrm{i}}(\mathrm{t}), \mathrm{i}=\overline{1,3}$, поддерживаются на своих заданных значениях $\theta_{i}^{3 \partial}(t)$ соответствующими регуляторами. При этом управляющие воздействия формируются на основе ошибок регулирования, в соответствии с выбранными для регуляторов алгоритмами регулирования:

$$
u_{н i}(t)=k_{p \theta i}\left(\Delta \theta_{i}(t)+\frac{1}{T_{u з} \theta_{i}} \int_{0}^{t} \Delta \theta_{i}(t) d t+T_{n p \theta i} \frac{d \theta_{i}(t)}{d t}\right)+u_{н i 0}, \quad \mathrm{i}=\overline{1,3}
$$

где $\mathrm{u}_{\text {ні0 }}$ - начальные значения управляющих воздействий $\mathrm{u}_{\text {ні }}(\mathrm{t})$ в момент замыкания контура регулирования, обеспечивающее «безударность» этого замыкания;

$\Delta \theta_{\mathrm{i}}(\mathrm{t})=\theta_{i}^{3 \partial}(t)-\theta_{\mathrm{i}}(\mathrm{t})-$ ошибки регулирования температур $\theta_{\mathrm{i}}(\mathrm{t})$;

$k_{p \theta_{i}}, T_{u з} \theta_{i}, T_{n p \theta_{i}}, \mathrm{i}=\overline{1,3},-$ настроечные параметры регуляторов температур, подлежащие оптимизации.

Оптимизация осуществлялась, как и в предыдущем случае, по интегральному квадратичному критерию в соответствии с выражением (1.4), в ходе специального компьютерного эксперимента с использованием

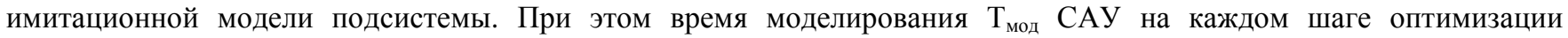
соответствовало времени, на котором осуществлялось моделирование возмущений, приведенных к каналам 
изменения напряжения питающей сети и влажности экструдируемого сырья.

$$
k_{p \theta_{i}}^{*}, T_{u з \theta_{i}}^{*}, T_{n p \theta_{i}}^{*}=\operatorname{argmin}\left\{\sum_{i=1}^{i=3} \int_{0}^{T_{\text {мо } о}}\left(\theta_{i}^{3 \partial}-\theta_{i}\left(k_{p \theta_{i}} T_{u з} \theta_{i}, T_{n p \theta_{i}}, t\right)\right)^{2} d t\right\},
$$

Значения начальных приближений настроечных параметров $k_{p \theta_{i}}, T_{u з} \theta_{i}, T_{n p \theta_{i}}, \mathrm{i}=\overline{1,3}$, для реализации первого шага поисковой процедуры оптимизации (1.4) найдены исходя из свойств типовых моделей динамики по каналу регулирования. При этом были приняты во внимание следующие две важные особенности «тепловой» составляющей ОУ. Первая. Величина времени запаздывания в указанных моделях несоизмеримо меньше, чем их постоянная времени, что облегчает достижение высокого качества регулирования. Вторая. Каналы регулирования температуры имеют перекрестные связи с коэффициентами передачи, величина которых соизмерима со значениями коэффициентов передачи в прямых каналах, что делает процесс регулирования температур взаимосвязанным и существенно затрудняет достижение высокого качества регулирования. С учетом рекомендаций [4 - 6] и сделанных выше замечаний, начальные значения настроечных параметров регуляторов приняты одинаковыми и равными: $k_{p}^{H} \theta_{i}$ $=10 \%$ у.в. ${ }^{0} \mathrm{C}, T_{u з}^{\mu} \theta_{\mathrm{i}}=3 \mathrm{c}, T_{n p \theta_{\mathrm{i}}}^{\mu}=1$ с. Значения параметров, найденные в ходе процедуры оптимизации и округленные, также приняты равными для всех трех регуляторов температуры: $k_{p}^{*} \theta_{i}=2,0 \%$ у.в. ${ }^{0} \mathrm{C}, T_{u з}^{*} \theta_{\mathrm{i}}=10 \mathrm{c}$, $T_{n p \theta_{\mathrm{i}}}^{*}=2 \mathrm{c}, \mathrm{i}=\overline{1,3}$.

Существенным недостатком классической структуры подсистемы регулирования температурного режима обработки сырья в экструдере, является то, что температура головки $\theta_{г}$ экструдера не регулируется. Ресурсы для такого регулирования были утеряны при отказе в базовой САУ от непрерывного изменения и в качестве управляющего воздействия САУ. Реализация структуры подсистемы регулирования температурного режима по «перекрестной» схеме, дает определенные ресурсы для регулирования $\theta_{\text {г }}$ Как и в предыдущем варианте, функции регулирования температур реализуются на основе замкнутого принципа управления, но, очевидно, имеют особенность, обусловленную перекрестной схемой регулирования. Ее сущность состоит в том, что управляющие воздействия і-той зоны формируются на основе ошибок регулирования температуры в i+1-ой зоне, причем температура в 4-ой зоне соответствует $\theta_{\text {г }}$ Для типовых алгоритмов регулирования выражения для формирования управляющих воздействий имеют вид:

$$
\begin{gathered}
u_{\mu i}(t)=k_{p \theta_{i+1}}\left(\Delta \theta_{i+1}(t)+\frac{1}{T_{u 3} \theta_{i+1}} \int_{0}^{t} \Delta \theta_{i+1}(t) d t+T_{n p \theta_{i+1}} \frac{d \theta_{i+1}(t)}{d t}\right)+u_{H i 0} \\
\mathrm{i}=\overline{1,3}, \quad \theta_{3+1}=\theta_{4} \equiv \theta_{\mathrm{r}}
\end{gathered}
$$

где $\Delta \theta_{i+1}(t)=\theta_{i+1}^{3 \partial}(t)-\theta_{i+1}(t), \theta_{4}^{3 \partial} \equiv \theta_{2}^{3 \partial}-$ ошибки регулирования температур $\theta_{\mathrm{i}+1}(\mathrm{t})$.

Результаты параметрического синтеза для алгоритмов (1.5) по критерию (1.6):

$$
\begin{gathered}
k_{p \theta_{i+1}}^{*}, T_{u 3 \theta_{i+1}}^{*}, T_{n p \theta_{i+1}}^{*}= \\
=\operatorname{argmin}\left\{\sum_{i=1}^{i=3} \int_{0}^{T_{\text {мо } \partial}}\left(\theta_{i+1}^{3 \partial}-\theta_{i+1}\left(k_{p \theta_{i+1}} T_{u 3 \theta_{i+1}}, T_{n p \theta_{i+1}}, t\right)\right)^{2} d t\right\}
\end{gathered}
$$

показали возможность сохранения настроек, которые были получены для предыдущего варианта структуры этой подсистемы.

Еще один вариант структуры подсистемы регулирования температурного режима обработки сырья, дающий возможность регулирования $\theta_{\text {г }}$, имеет каскадную структуру, где главной регулируемой переменной является

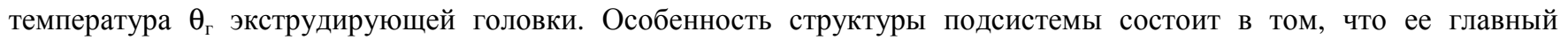
регулятор (регулятор $\theta_{\text {г }}$ ) устанавливает одновременно заданные значения сразу трем вспомогательным регуляторам 
(регуляторам $\theta_{\mathrm{i}}, \mathrm{i}=\overline{1,3}$ ), а не одному, как в классической каскадной структуре. Для типовых алгоритмов регулирования выражения для формирования управляющих воздействий вспомогательных и главного регулятора имеют вид:

$$
\begin{aligned}
& u_{\mu i}(t)=k_{p \theta i}\left(\Delta \theta_{i}(t)+\frac{1}{T_{u з} \theta i} \int_{0}^{t} \Delta \theta_{i}(t) d t+T_{n p \theta i} \frac{d \theta_{i}(t)}{d t}\right)+u_{\mu i 0}, \quad \mathrm{i}=\overline{1,3},
\end{aligned}
$$

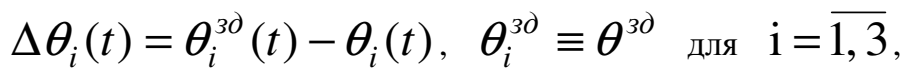

$$
\begin{aligned}
& \theta^{3 \partial}(t)=k_{p \theta_{2}}\left(\Delta \theta_{2}(t)+\frac{1}{T_{\text {из } \theta_{2}}} \int_{0}^{t} \Delta \theta_{2}(t) d t+T_{n p \theta_{2}} \frac{d \theta_{2}(t)}{d t}\right)+\theta_{20}^{3 \partial} \\
& \Delta \theta_{2}(t)=\theta_{2}^{3 \partial}(t)-\theta_{2}(t) .
\end{aligned}
$$

Для этого варианта подсистемы настройки вспомогательных регуляторов температуры были сохранены на значениях, принятых после оптимизации параметров регуляторов температуры. Для рассматриваемого варианта подсистемы параметрический синтез проводился только для главного регулятора, в соответствии с критерием:

$$
\begin{gathered}
k_{p \theta_{2}}^{*}, T_{u 3 \theta_{2}}^{*}, T_{n p \theta_{2}}^{*}= \\
=\operatorname{argmin}\left\{\int_{0}^{T \mu o \partial \partial}\left(\theta_{2}^{3 \partial}(t)-\theta_{2}\left(k_{p \theta_{2}} T_{u 3 \theta_{2}}, T_{n p \theta_{2}}, t\right)\right)^{2} d t\right\},
\end{gathered}
$$

Принятые значения этих настроек: $k_{p \theta_{2}}^{*}=1,0 \%$ у.в. $/^{0} \mathrm{C}, T_{u з}^{*} \theta_{2}=15 \mathrm{c}, T_{n p \theta_{2}}^{*}=3 \mathrm{c}$.

Анализ работы подсистем регулирования с типовыми алгоритмами. Анализ работы подсистемы регулирования тока нагрузки ПЭД экструдера и альтернативных вариантов подсистемы регулирования температурного режима обработки сырья в экструдере проводился на полной имитационной модели ОУ и действующих на него возмущений. Время моделирования $\mathrm{T}_{\text {мод }}$ соответствовало времени, на котором осуществлялось моделирование возмущений, приведенных к каналам изменения напряжения питающей сети и влажности экструдируемого сырья.

В качестве базы для сравнения, первоначально, на этом же интервале времени, было проведено моделирование работы объекта при фиксированных управляющих воздействиях $\mathrm{u}_{\ni}=0,75, \mathrm{u}_{\mathrm{n}}=17,2 \%$ у.в, $\mathrm{u}_{\mathrm{H} 1}=20 \%$ у.в, $\mathrm{u}_{\mathrm{H} 2}=30 \%$ у.в, $\mathrm{u}_{\mathrm{H} 3}=45 \%$ у.в, значения которых были подобраны специальным образом. Основными требованиями при этом подборе было обеспечение температурного режима обработки сырья в экструдере в окрестности регламентированных значений и максимально возможная подача сырья в экструдер (производительность экструдера), при которой на интервале моделирования не происходило бы срабатывания тепловой защиты ПЭД экструдера из-за его перегрузки. Такой вариант управления процессом можно рассматривать как вариант управления оператором («ручного» управления процессом). Результаты его моделирования приведены на рис. 1, а моделирования, подтверждающего предельную загрузку экструдера сырьем для этого варианта, на рис. 2. Анализ рис. 1 показывает, что все регламентированные переменные процесса экструдирования под действием возмущений существенно изменяются и, безусловно, задачи и автоматического регулирования температурного режима обработки сырья при экструдировании и тока нагрузки ПЭД экструдера весьма актуальны. 


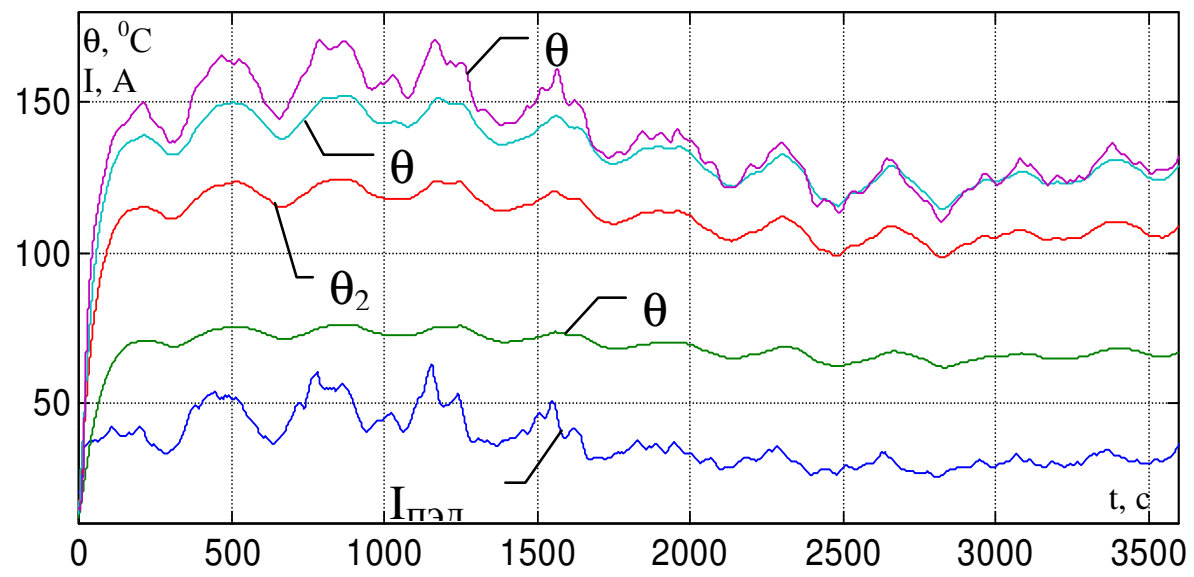

Рис. 1. Моделирование процесса экструдирования при

фиксипованных vпnавпяютих впзлействиях :

$\mathrm{u}_{3}=0,75, \mathrm{u}_{\mathrm{n}}=17, \%$ у.в., $\mathrm{u}_{\mathrm{H} 1}=20 \%$ у.в., $\mathrm{u}_{\mathrm{H} 2}=30 \%$ у.в., $\mathrm{u}_{\mathrm{H} 3}=45 \%$ у.в.,

$\left(\mathrm{u}_{\text {п }}=17,2 \%\right.$ у.в. соответствует максимуму производительности экструдера без перегрева его ПЭД на интервале времени $\mathrm{T}_{\text {мод }}$ )

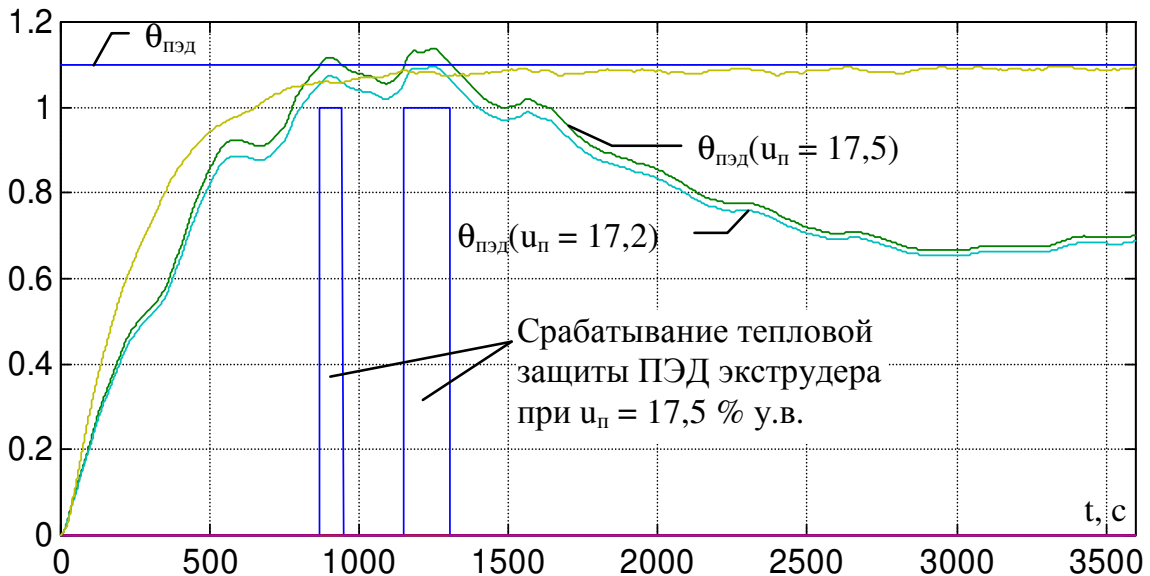

Рис. 2. Результаты моделирования, подтверждающего предельную

загрузку экструдера сырьем при $\mathrm{u}_{\Pi}=17,2 \%$ у.в.

Для оценки эффективности разработанных систем управления приводятся результаты моделирования двух «промежуточных» ситуаций, когда реализована одна из двух подсистем регулирования, либо температурного режима обработки сырья, см. рис. 3, либо тока нагрузки ПЭД, см. рис. 4. В первой ситуации, при стабилизации температур по зонам, их заданные значения достаточно точно выдерживаются за счет изменения соответствующих управляющих воздействий. При этом температура экструзионной головки существенно уменьшила размах своих колебаний, но она сохранила свою зависимость от степени нагрузки на ПЭД экструдера, которая, в том числе, пропорциональна механической работе, выполняемой над сырьем, и, следовательно, его нагреву за счет этой работы. Во второй ситуации стабилизация тока нагрузки ПЭД за счет изменения подачи сырья на экструдирование одновременно позволяет существенно снизить размах колебаний температур и в зонах нагрева и в экструдирующей головке. Этот факт указывает на то, что в условиях минимизации затрат на автоматизацию экструдера, целесообразно в первую очередь решать задачу автоматической стабилизации тока нагрузки его ПЭД. 

ТЕХНОЛОГІЧНИМИ ПРОЦЕСАМИ

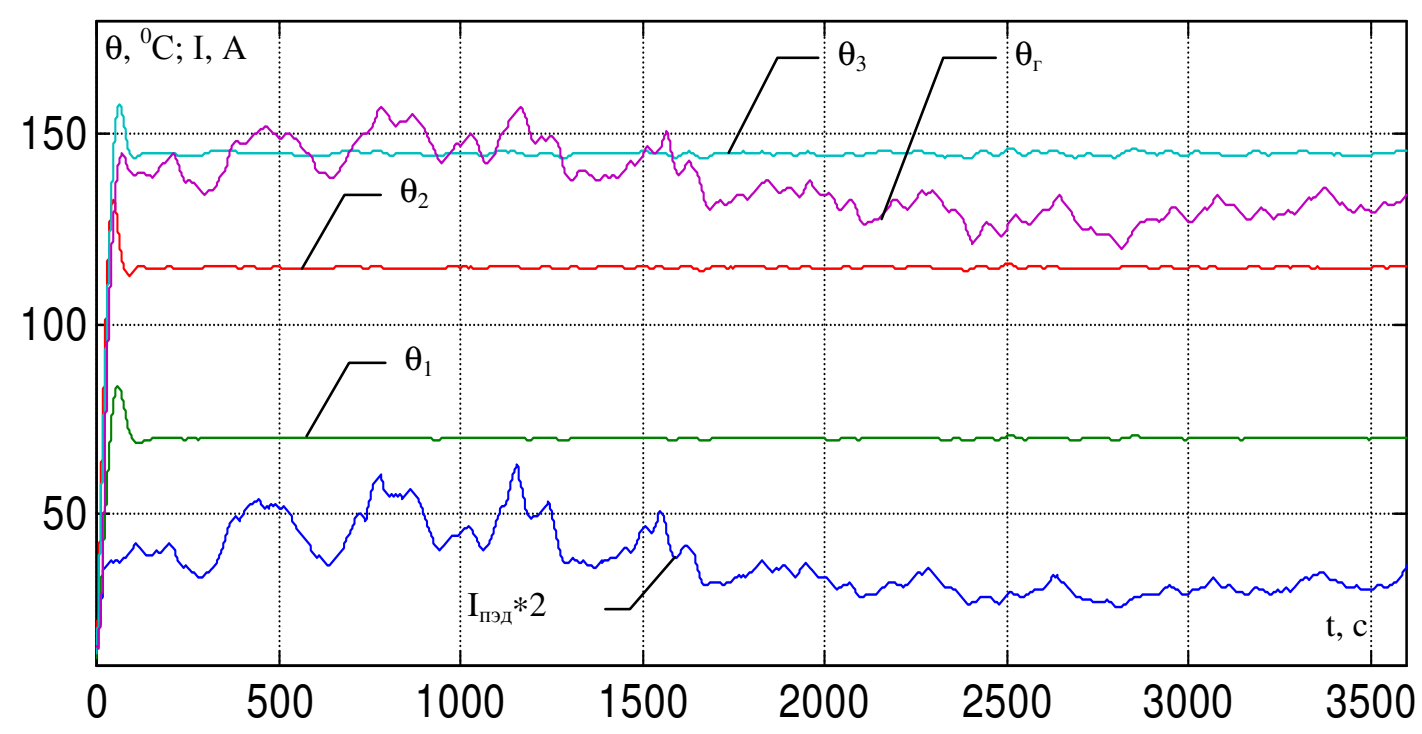

a)

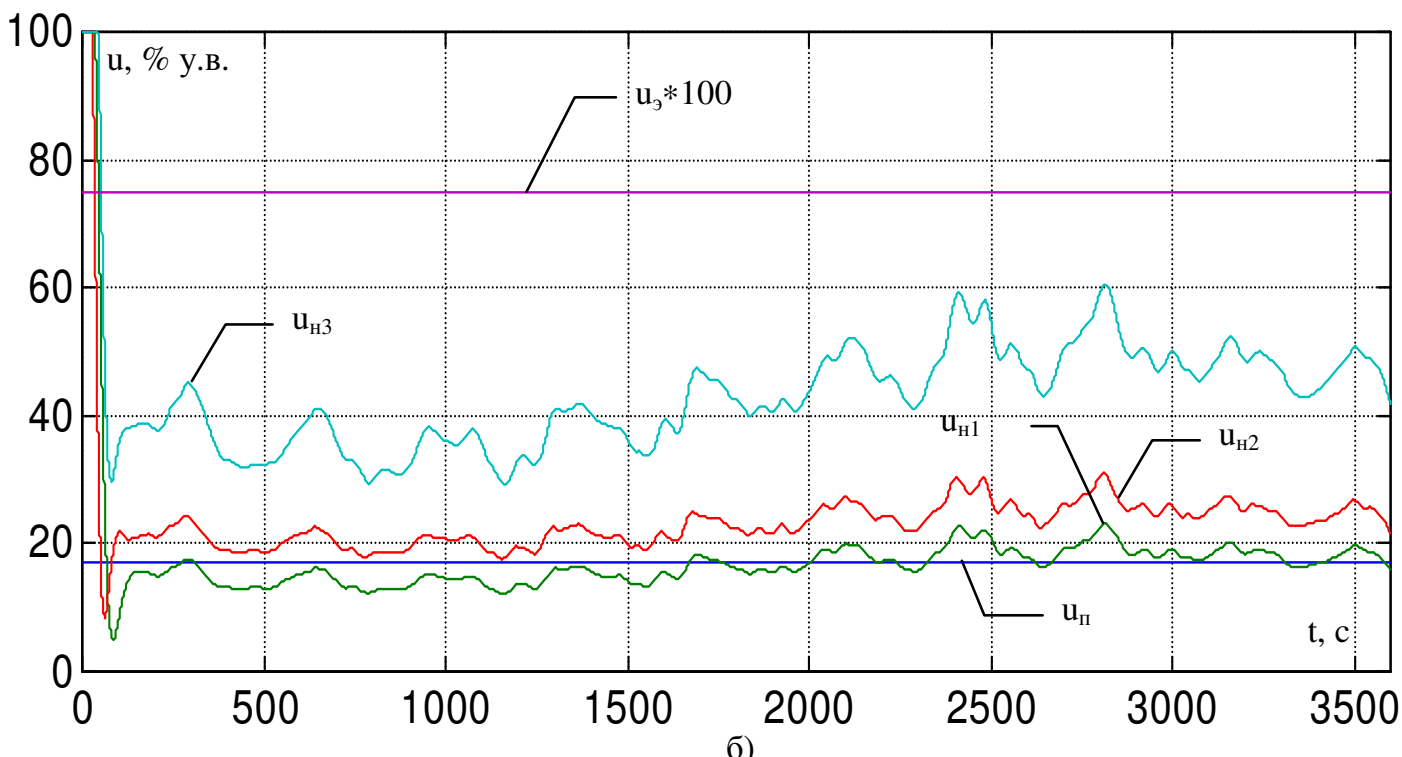

Рис. 3. Моделирование процесса экструдирования при автоматическом регулировании температурного режима обработки сырья для $\theta_{1}^{3 \partial}=70{ }^{0} \mathrm{C}$, $\theta_{2}^{3 \partial}=115^{0} \mathrm{C}, \theta_{3}^{3 \partial}=145{ }^{0} \mathrm{C}$, и фиксированных управляющих воздействиях: $\mathrm{u}_{3}=0,75, \mathrm{u}_{\text {п }}=17,2 \%$ у.в., где: а) изменение регламентированных переменных; б) изменение управляющих воздействий 

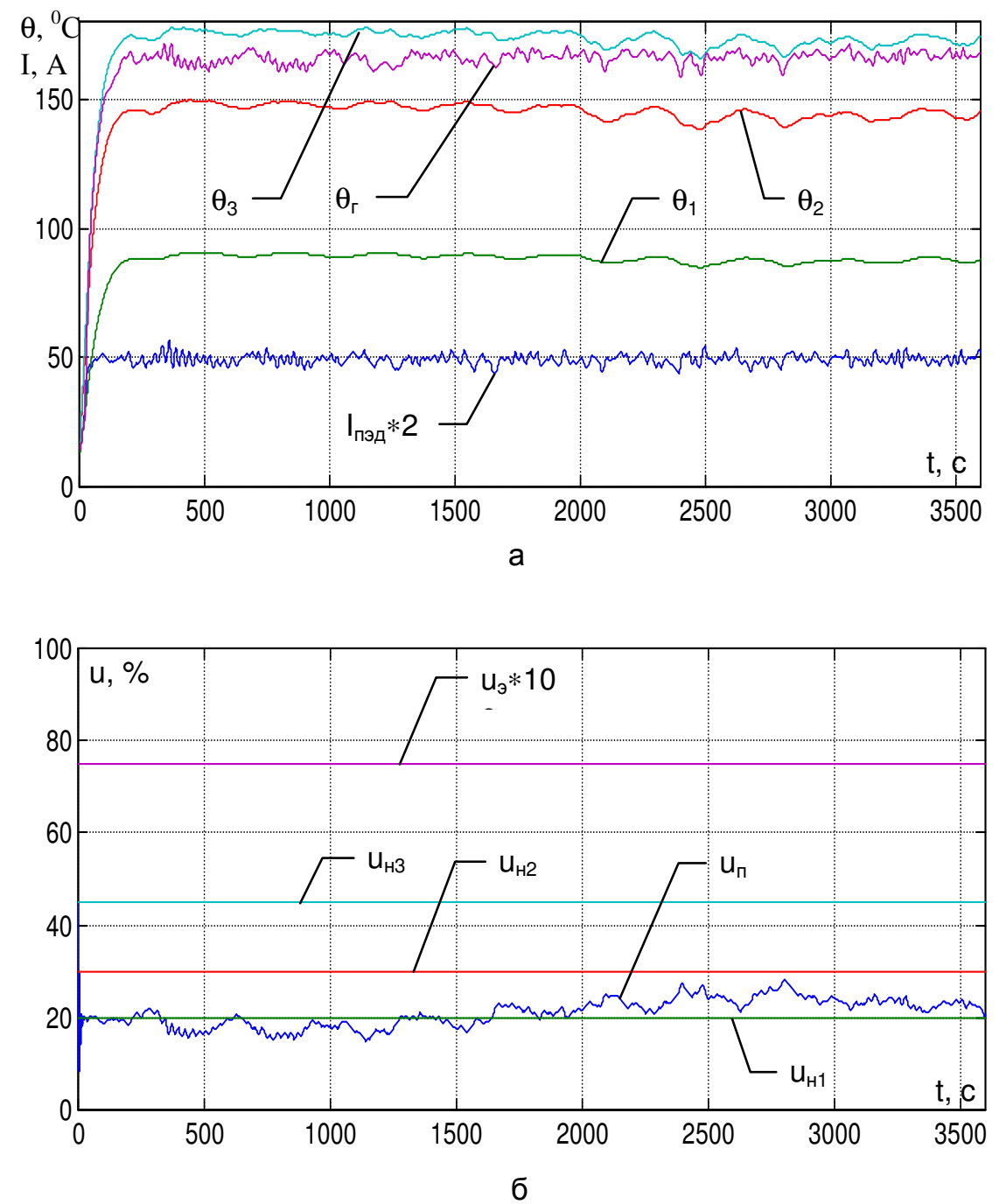

Рис. 4. Моделирование процесса экструдирования при автоматическом регулировании тока нагрузки ПЭД при $I_{n \ni \partial}^{3 \partial}=24,6 \mathrm{~A}\left(I_{n \ni \partial}^{3 \partial}=24,6 \mathrm{~A}\right.$, соответствует максимуму производительности экструдера без перегрева его ПЭД на интервале времени $\mathrm{T}_{\text {мод}}$ ), для фиксированных управляющих воздействий: $\mathrm{u}_{3}=0,75, \mathrm{u}_{\mathrm{H} 1}=20 \%$ у.в., $\mathrm{u}_{\mathrm{H} 2}=30 \%$ у.в., $\mathrm{u}_{\mathrm{H} 3}=45 \%$ у.в., где: а) изменение регламентированных переменных; б) изменение управляющих воздействий

Схема моделирования САУ процессом ЭБП при автоматическом регулировании тока нагрузки ПЭД, $\mathrm{I}^{\text {3д }}=24,6$ А, и температурного режима обработки сырья, вариант прямого регулирования температур, $\theta_{1}^{3 \partial}=70{ }^{0} \mathrm{C}, \theta_{2}^{3 \partial}=115{ }^{0} \mathrm{C}$, $\theta_{3}^{3 \partial}=145{ }^{0} \mathrm{C}$, для фиксированного управляющего воздействия и моделирования - на рис 6. Как следует из этого рисунка, тепла, которое выделяется при выполнении механической работы над сырьем при такой его подаче, которая соответствует заданному значению тока нагрузки ПЭД экструдера $\mathrm{I}^{3 д}=24,6$ А, достаточно, чтобы удерживать температуры в зонах нагрева выше своих заданных значений без подвода тепла от ТЭНов. Именно поэтому, после окончания переходных процессов в контурах регулирования температур $\theta_{1}$, $\theta_{2}$ и $\theta_{3}$ все их управляющие воздействия $\mathrm{u}_{\mathrm{H} 1}, \mathrm{u}_{\mathrm{H} 2}, \mathrm{u}_{\mathrm{H} 3}$ стали равными нулю.

Схема моделирования САУ процессом ЭБП при автоматическом регулировании тока нагрузки ПЭД, $\mathrm{I}^{3 д}=24,6$ А, 
и температурного режима обработки сырья, вариант перекрестного регулирования температур, $\theta_{2}^{3 \partial}=115^{0} \mathrm{C}, \theta_{3}^{3 \partial}=$

$145{ }^{0} \mathrm{C}, \theta_{2}^{3 \partial}=150{ }^{0} \mathrm{C}$, для фиксированного управляющего воздействия $\mathrm{u}_{\ni}=0,75$, приведена на рис. 7 , а результаты этого моделирования - на рис 8. Как следует из этого рисунка, при такой структуре подсистемы автоматического регулирования температурного режима обработки сырья появляется возможность целенаправленно влиять на

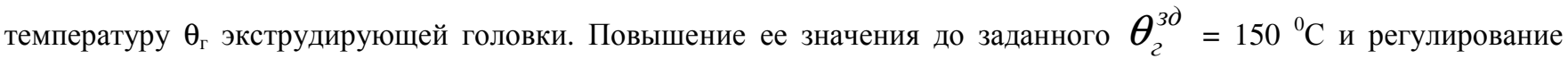
(стабилизация) на заданном значении осуществляется, в данном примере, за счет управляющего воздействия $\mathrm{u}_{\mathrm{H} 3}$. Это, одновременно, вызывает рост температур в соседних зонах нагрева выше заданных значений даже при нулевых значениях $\mathrm{u}_{\mathrm{H} 1}$ и $\mathrm{u}_{\mathrm{H} 2}$. Последствия такого перегрева в рамках данной работы оценить невозможно. Они могут быть как отрицательными, так и положительными, причем в зависимости от вида экструдируемого сырья и его характеристик. При всем этом необходимо учитывать, что указанные значения температур соответствуют температурам корпуса экструдера в зонах прессования, а не температурам перемещающегося через эти зоны продукта.

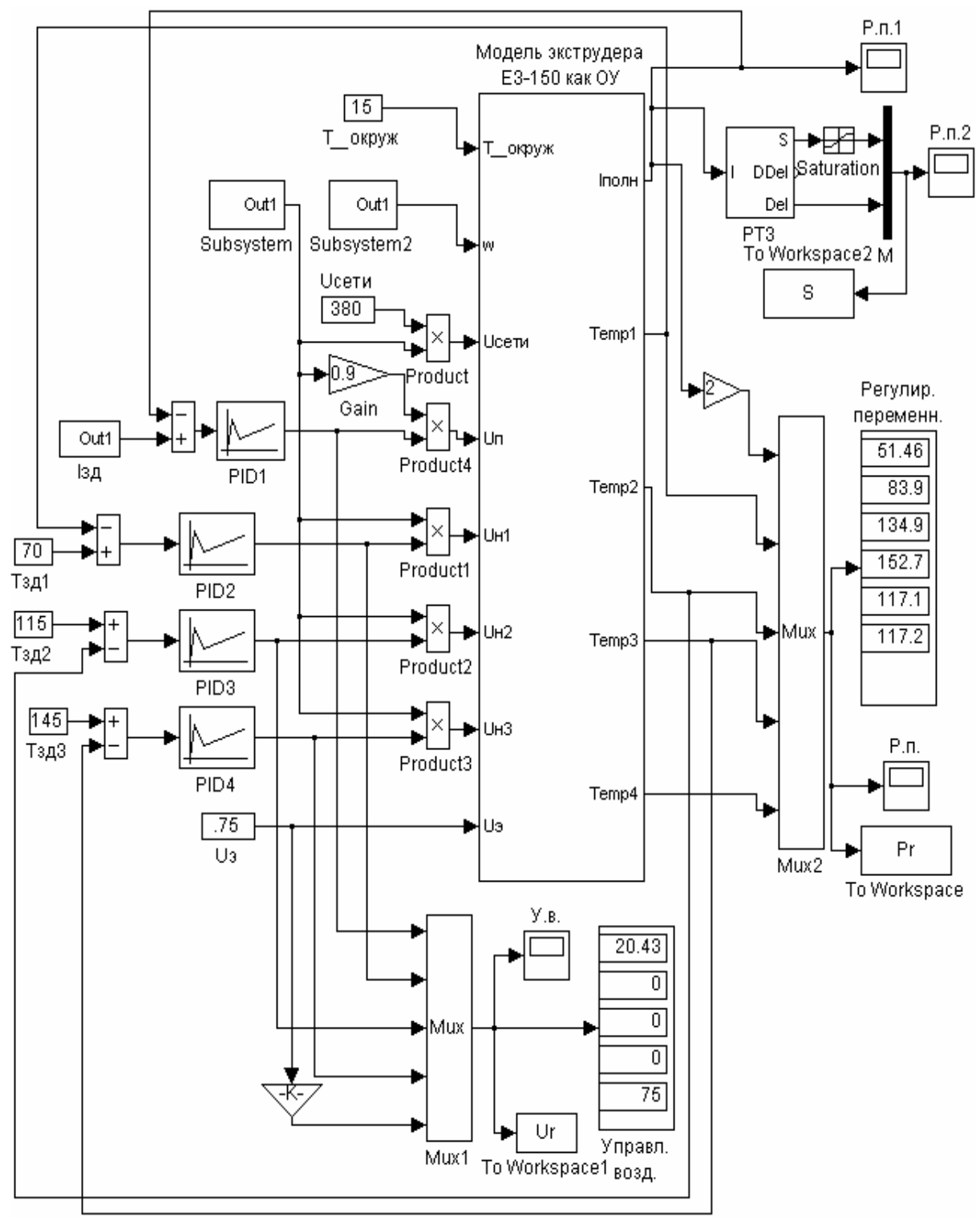

Рис. 5. Схема моделирования САУ процессом ЭБП при автоматическом регулировании тока нагрузки ПЭД и температурного режима обработки сырья, вариант прямого регулирования температур, для фиксированного управляющего воздействия $\mathrm{u}_{\ni}=0,75$ 


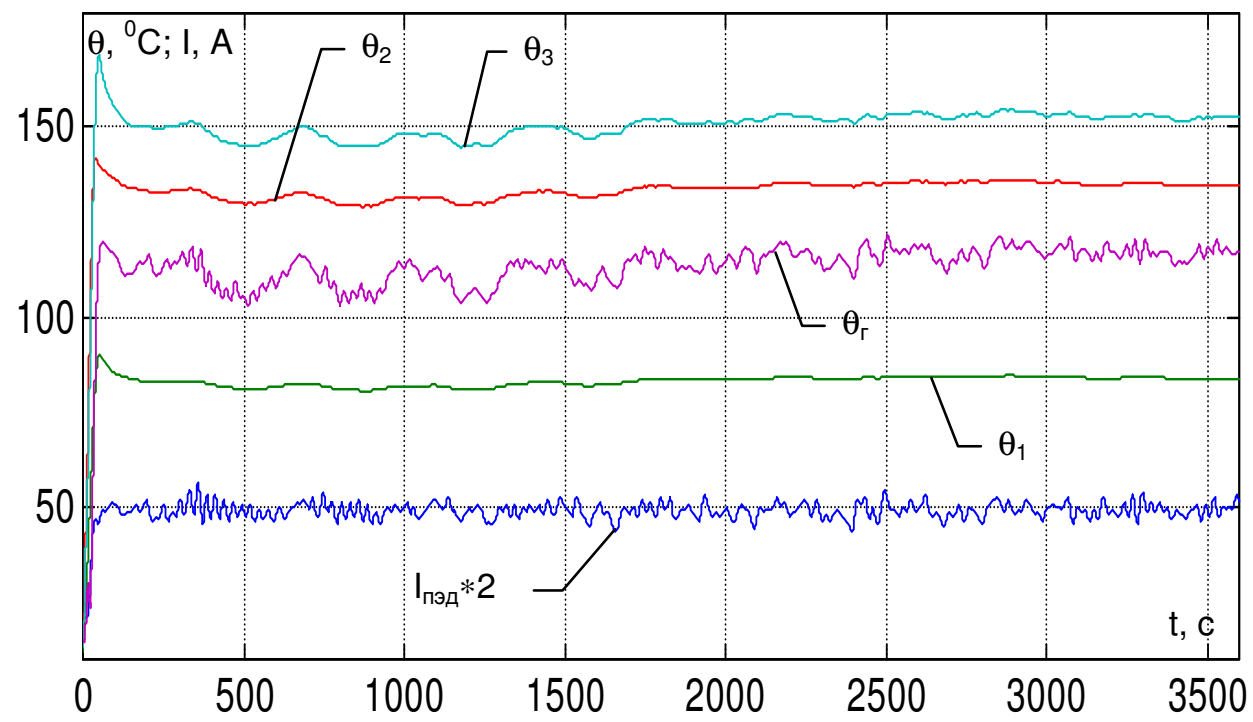

a)

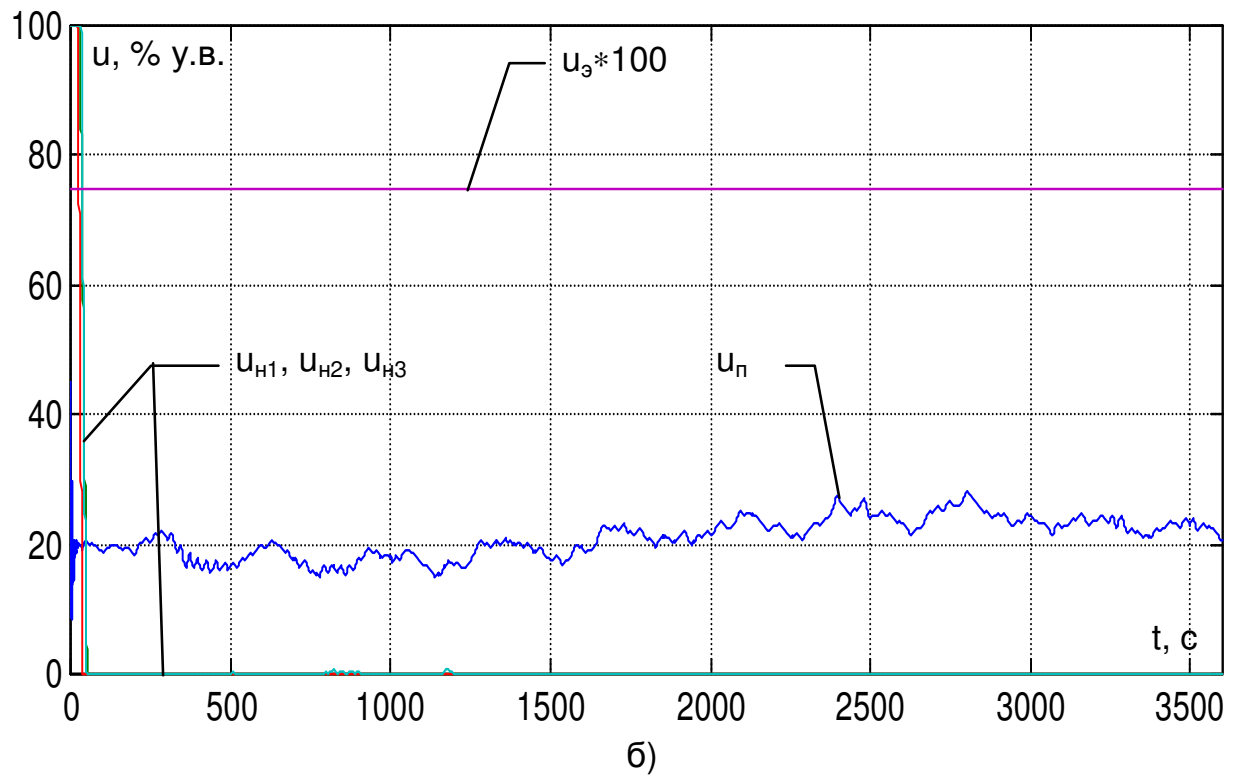

Рис. 6. Моделирование процесса ЭБП при автоматическом регулировании тока нагрузки ПЭД, $I_{n \ni д ~}^{3 d}=24,6$ А, и температурного режима обработки сырья (вариант прямого регулирования температур), $\theta_{1}^{3 \partial}=70{ }^{\circ} \mathrm{C}, \theta_{2}^{3 \partial}=115^{\circ} \mathrm{C}, \theta_{3}^{3 \partial}=145^{\circ} \mathrm{C}$, для фиксированного управляющего воздействия $\mathrm{u}_{3}=0,75$, где: а) изменение регламентированных переменных;

б) изменение управляющих воздействий 


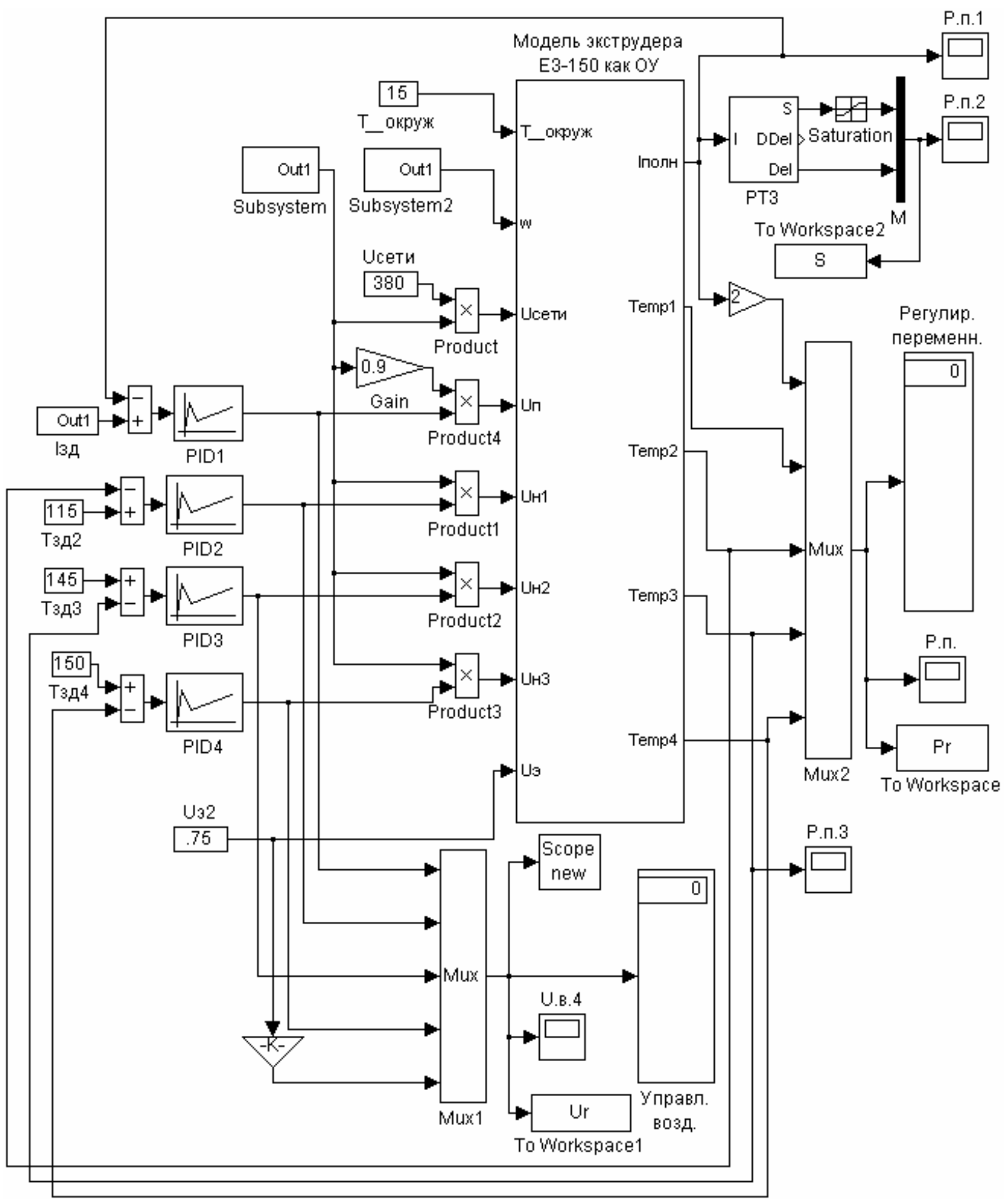

Рис. 7. Схема моделирования САУ процессом ЭБП при автоматическом регулировании тока нагрузки ПЭД и температурного режима обработки сырья, вариант перекрестного регулирования температур, для фиксированного управляющего воздействия $\mathrm{u}_{\ni}=0,75$ 


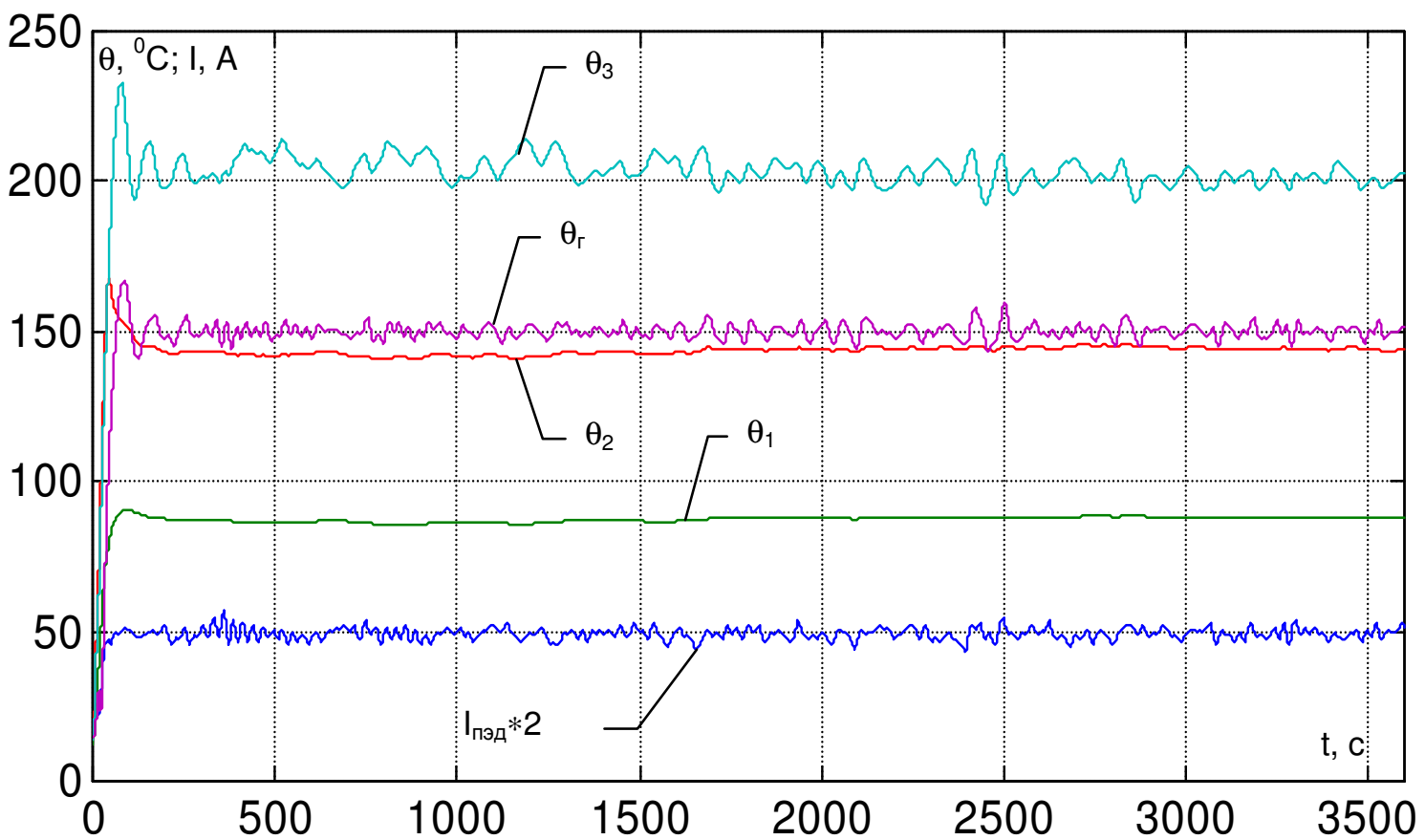

a)

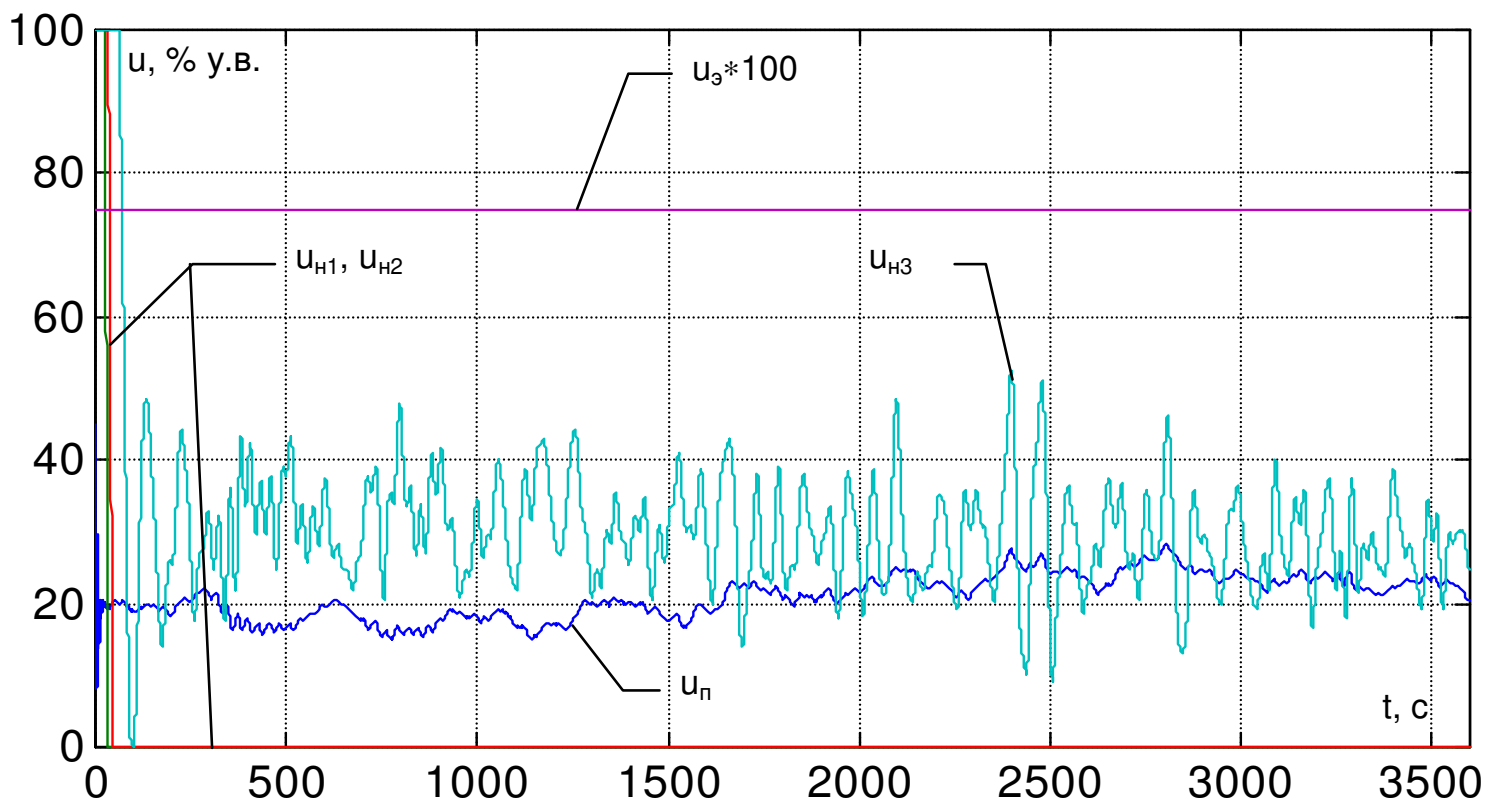

б)

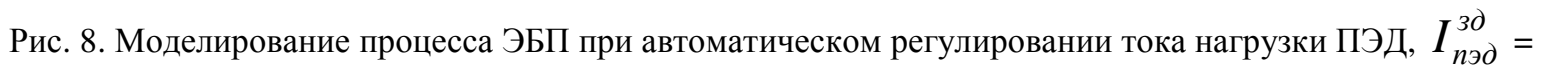
24,6 А, и температурного режима обработки сырья (вариант перекрестного регулирования температур), $\theta_{2}^{3 \partial}=115{ }^{0} \mathrm{C}, \theta_{3}^{3 \partial}=145{ }^{0} \mathrm{C}, \theta_{2}^{3 \partial}=150{ }^{\circ} \mathrm{C}$, для фиксированного управляющего воздействия $\mathrm{u}_{\ni}=0,75$, где: а) изменение регламентированных переменных; б) изменение управляющих воздействий 
Выводы: Для разработки (синтеза и анализа) и реализации действующего варианта САУ процесса ЭБП было введено понятие ее базового варианта. Он ориентирован решать в автоматическом режиме наиболее динамичные задачи управления, сохранив за оператором и лабораторией управление качеством экструдатов. Разработка САУ, реализующих эти задачи, сделала необходимым проведение структурного и параметрического синтеза системы, анализа ее функционирования, адекватно отражающего условия ее будущей эксплуатации.

Процедура синтеза САУ отражала, в определенной мере, ее иерархическую структуру. Первоначально, для нижнего иерархического уровня, уровня регулирования, был проведен структурный и параметрический синтез систем регулирования, ориентированный на применение типовых алгоритмов. Синтез подсистемы регулирования температурного режима обработки сырья включал три альтернативных варианта. При этом все три варианта, имея свои достоинства, показали в ходе их анализа достаточно хорошую динамическую точность с типовыми алгоритмами для всех условий моделирования. Это сняло вопрос о необходимости совершенствования структуры системы регулирования и/или ее алгоритмов.

\section{Литература}

1. Рей У. Методы управления технологическими процессами: Пер. с англ. - М.: Мир, 1983. - 368 с.

2. Бессекерский В.А., Небылов А.В. Робастные системы автоматического управления. - М.: Наука, 1983. - 240 c.

3. Бесекерский В.А, Изранцев В.В. Системы автоматического управления с микроЭВМ: Теоретические основы технической кибернетики. - М.: Наука, 1987. - 320 с.

4. Наладка средств автоматизации и автоматических систем регулирования: Справочное пособие / Под ред. А.С. Клюева. - 2-е изд. - М.: Энергоатомиздат, 1989. - 368 с.

5. Широкий Д.К., Куриленко О.Д. Расчет параметров промышленных систем регулирования / Справочное пособие. - К.: «Техніка», 1972. - 232 с.

6. Трегуб В.Г., Ладанюк А.П. Проектирование, монтаж и эксплуатация систем автоматизации пищевых производств. - М.: Легкая и пищ. пром-сть, 1980. - 352 с.

7. Хобин В.А., Егоров В.Б. Проблемы математического описания процесса экструдирования биополимеров как объекта управления и технология их преодоления // Сб. докл. III Всеукр. науч.-практ. конф. «Информационные технологии и автоматизация - 2010». - Одесса: ОНАПТ, 2010. - С. 61 - 62.

8. Хобин В.А., Егоров В.Б. Имитационная математическая модель процесса экструдирования биополимеров как объекта управления // Наук. пр. ОНАХТ / Міністерство освіти України. - Одеса: 2010. - Вип. 38. - Т. 1. - С. $386-399$.

9. Кругликов В.К. Вероятностный машинный эксперимент в приборостроении. - Л.: Машиностроение: Ленингр. отд-ние, 1985. - 247 с.: ил.

10. Математическое обеспечение системы ЭВМ - экспериментатор. - М.: Наука, 1977. - 251 с.

11. Смит Джон М. Математическое и цифровое моделирование для инженеров и исследователей: Пер. с англ. М.: Машиностроение, 1980. - 271 с.

12. Ажогин В.В., Згуровский М.З. Моделирование на цифровых, аналоговых и гибридных ЭВМ. - К.: Вищ. шк., 1983. $-280 \mathrm{c}$. 